\title{
Factors Affecting Health Seeking Behaviors Among Mothers of Under Five Children Attending Primary Health Care Centers, Sudan
}

\author{
Mahmouda Mohammed Khalil ${ }^{1, ~ *}$, Elfatih Mohamed Malik ${ }^{2}$, Nada Mohamed Ali Alnair ${ }^{3}$, \\ Ibrahim Ismail Mohamed Abu ${ }^{4,5}$ \\ ${ }^{1}$ Health Information and M \& E Directorate, Federal Ministry of Health, Khartoum, Sudan \\ ${ }^{2}$ Community Medicine Department, University of Khartoum, Khartoum, Sudan \\ ${ }^{3}$ Academy of Health Sciences, Federal Ministry of Health, Khartoum, Sudan \\ ${ }^{4}$ Community Medicine Department, King Abdul-Aziz University, Jedha, Kingdom of Saudi Arabia \\ ${ }^{5}$ Community Medicine Department, Al Fashir University, Al Fashir, Sudan

\section{Email address:} \\ hamdo016@yahoo.com (M. M. Khalil), fatihmmalik@hotmail.com (E. M. Malik), nadaalnayer@gmail.com (N. M. A. Alnair), \\ burha75@gmail.com (I. I. M. Abu) \\ ${ }^{*}$ Corresponding author
}

\section{To cite this article:}

Mahmouda Mohammed Khalil, Elfatih Mohamed Malik, Nada Mohamed Ali Alnair, Ibrahim Ismail Mohamed Abu. Factors Affecting Health Seeking Behaviors Among Mothers of Under Five Children Attending Primary Health Care Centers, Sudan. Science Journal of Public Health. Vol. 7, No. 6, 2019, pp. 181-186. doi: 10.11648/j.sjph.20190706.11

Received: September 24, 2019; Accepted: October 18, 2019; Published: October 30, 2019

\begin{abstract}
Children represent the most vulnerable age group in any community. In Sudan they have higher morbidity and mortality rates. Mothers' health seeking behaviors are major determinants for improving child health and knowledge about the factors influencing such practice is very crucial for designing effective intervention strategies. This studyaimsto study factors affecting health seeking behaviors for illness in under five children among the mothers in Khartoum state, Sudan. It was Across-sectional study usingbothquantitative and qualitative methods, Four hundreds and ninety five (495) mothers of those who visited the vaccination units of 33 public health centers were interviewed. Four focus group discussions were held. Chisquare test and regression analysis were used to determine the association between different factors and health seeking behaviors. The results showed that Eighty percent of mothers $(80 \%)$ seek health care for their ill children. Of them, $70 \%$ went immediately to heath facility after disease occurrence. The person who took the decision of going to health facility ( $p$ value $=0.000$ ) and work time for the health facility ( $p$-value $=0.001)$ were found to be significantly associated with mothers' seeking behavior using bivariate and multivariate analysis. Since the person who took the decision of going to health facility is the most determinant for mothers' seeking behavior, awareness rising among mothers about the importance of going to health facility immediately after disease occurrence can improve child health outcomes.
\end{abstract}

Keywords: Children, Mothers, Health Seeking Behavior, Treatment Seeking, Behavior

\section{Introduction}

Children represent the majority of vulnerable age group in any community. The child mortality rate is widely used as a demographic evaluate and a fundamental indicator of the level of welfare in most countries. An estimated 5.4 million children under age 5 died in 2017; half of them occurred in sub-Saharan Africa. More than half of these deaths were due to preventable conditions where access to simple and affordable interventions was feasible [1].

Children under the age of 5years in Sudan represent $15 \%$ of the total population. The infant mortality was52/1000 live births while under-five mortality was68/1000 of live births [2]. Infant morbidity and mortality are due mainly to the persistence of infectious diseases and malnutrition [3]. The 2015 Sudan health statistical report showed that deaths 
among under five children were causedmostly by malaria $(17 \%)$, pneumonia $(14 \%)$, malnutrition $(13 \%)$ and diarrhoea (9\%) [4]. The World Health Organization (WHO) and United Nations Children's Fund (UNICEF) draw attention to the importance of seeking early care, and they developed the strategy called 'Integrated Management of Childhood Illness' (IMCI), which highlights appropriate family and community health practices are vital for improving the health status of children and declining childhood mortality in the majority of developing countries [5].

In Sudan the Child Health Policy and the IMCI program have been developed since 1997 and expanded to cover all the states at the health facility and community levels. Still the leading causes of death among children under 5-years have remained unchanged for several years. Causes for such unprogressive outcomes may include issues related to utilization of health services, quality of care, health seeking behaviors and other factors [6].

However, success in reducing childhood mortality needs more than the availability of adequate health services with well-trained health professionals. As families are the first people responsible for child care, success requires a partnership between health workers and families with community support [6]. Health-seeking behavior has been defined as a "sequence of remedial actions that individuals undertake to rectify perceived ill-health". In particular, healthseeking behavior can be described with data collected from information such as the time difference between the onset of an illness and getting in contact with a healthcare professional, type of healthcare provider patients sought help from, how the compliant of the patient is enlightened with the recommended treatment, reasons for choice of healthcare professional and reasons for not seeking help from healthcare professionals [7].

In Sudan many interventions are available in many places but many children cannot utilize them because of health seeking behavior of their mothers or care givers. Although mothers' health seeking behaviors are major determinants for improving child health, and knowledge about thefactors influencing such practice is very crucial for designing effective strategies for improving them, there is little evidence on the nature of such factors in the challenging context like Sudan.

\section{Material and methods}

\subsection{Study Setting}

This was cross-sectionalfacility-based study usingboth quantitative and qualitative methods, the study was conducted in Khartoum State. The State has a total population of $6,311,500$. There are 232 primary health care centres distributed over seven localities, in 2016 these centers have under five outpatient visits of 190,258.

\subsection{Study Population}

Mothers of under 5 children in Khartoum State who attended the vaccinationsessionat primary health centreswhomchildren suffered from at least one of the symptoms related to the IMCI targeted diseases in the previous month.

\subsection{Sample Size and Sampling}

Using the formula $\left(n=z^{2} \times p q / d^{2}\right)$ the sample size was calculated where $(\mathrm{n})$ is thesample size and $(\mathrm{z})$ isstandard normal deviate which equals to $(1.96),(p)$ is proportion of the level of health seeking behaviorestimated in mothers of children, while (q) equals to (1-p) and (d) is the error allowed which is (0.05) and with design effect equals to (1.5); this yields a sample of 495 mothers. Cluster random sampling technique was used. The cluster wasthe primary health care centre. The cluster size was 15 mothers, so a total of thirty three centers were chosen, distributed over the seven localities using probability proportional to size. The selection of the health centers and ultimatelythe 15 respondents was done using systematic random sampling.

\subsection{Data Collection}

Six trained data collectors obtained the data via interviewing mothers using structured, pre-tested and precoded questionnaire. For qualitative data, four focus group discussions were conducted by the trained team using focus group discussion guide with seven mothersin each recruited from the areas around the selected health centers.

\subsection{Data Management and Analysis}

Data was cleaned, entered and analyzed using SPSS version (20). Descriptive data presented as graphs, frequency tables. Inferential statistics was conducted using crosstabulation. Chi-square was obtained, and P-value less than (0.05) was considered significant. Logistic regression was conducted to identify the most significant factors. Qualitative analysis was done manuallyusingthematic analysis.

\subsection{Ethical Consideration}

This study was approved from the ethical and technical committee of Sudan Medical Specialization Board and Khartoum State Ministry of Health. Informed consent from each study participant was obtained prior the interview or focus group discussion.

\section{Results}

Among the 495 mothers interviewed, 67\% (330) of them are living in urban area and $76 \%$ (376) are housewives. Regarding mother' education, the study indicated that $38 \%$ (188) of mother are of secondary level of education while only $1.8 \%$ (9) are postgraduate. The study showed that about $62 \%$ (309) of mother lived in their own house, and two third of the interviewed mother have income more than 1000 Sudanese pound monthly. Lastly, only $23 \%$ (112) having a car (Table 1).

Approximately there is an equal distribution among age groups and sex of all children of the interviewed mothers. 
The study revealed that two third of children of interviewed mothers were either the first or the second child $32.7 \%$ (161) and $29.9 \%$ (146) respectively. Furthermore, the study indicated that two third of children have one sibling $(66.2 \%)$. Nearly all the children were full term babies (95.8\%-474) with only $8 \%$ (41) suffering from chronic diseases.

The study revealed that the majority $(80 \%)$ of interviewed mothers seek health for their ill children either by going to the health facility immediately $(70.3 \%-348)$ or went to health facility after 24 hours $(9.7 \%-48)$. Accordingly, the tow third of mothers $(33.3 \%-165)$ thought that the disease of their child was mild. On the other hand, the study indicated that parents took the decision of taking their ill children to the health facility by themselves $(71.3 \%-353)$. The study revealed that governmental health centers are the nearest health facility for the mothers $(83.8 \%-414)$ with time taken to reach it about 10 minutes in average. Most of mothers used to go to the health facility with local transport. The study indicated that approximately half of health facilities works for the whole day, with the services available in about 63\% (311) of them. Half of mothers thought that the quality of services at health facility is good (58.4\%-289), with 70\% (345) thought that the services cost is affordablefor them. Sixty percent (298) of children are covered with health insurance, and only $21 \%$ (102) of mothers affected by the tradition and culture negatively.

Bivariate analysis revealed that, eightfactors were found to have significant association with mothers, seeking behavior.
These are, residence $(p=0.032)$, income $(p=0.030)$, child age $(p=0.006)$, quality of services at the health facility $(p=0.005)$, the person who took the decision of going to the facility $(p=0.0001)$, work time for health facility $(p=0.018)$, health insurance availability for children $(\mathrm{p}=0.031)$, and tradition and customs $(p=0.0001)$. While level of education $(p=0.676)$, occupation $(\mathrm{p}=0.126)$ ownership of car $(\mathrm{p}=0.485)$, child order $(p=0.088)$, child sex $(p=0.472)$, illness severity $(p=0.491)$, cost of services $(p=0.463)$ and availability of services $(p=0.177)$ found to have no relation with seeking health in this study.

Logistic regression showed that only six factors were significantly related to seeking health. The person who took the decision of going to the health facility was the most predictor factor for seeking care $(\mathrm{OR}=1.755$; 95\% CI: 1292 2383). The second predictor was found to be the work time of health facility $(\mathrm{OR}=0.415 ; 95 \% \mathrm{CI}: 0.241-0.713)$ followed by tradition and culture $(\mathrm{OR}=0.451 ; 95 \% \mathrm{CI}$ : $0.258-0.790)$, which affect mother' seeking behavior negatively.

Focus group discussion reflectedthat, most of mothers mentioned that when their children get ill first they start with home treatment by traditional medicine and cold sponge. Then if there is no improvement or the disease becomes severe they go to the nearest health facility, and the above factors have strong effect on them especially when the disease is severe. Approximately all the mothers mentioned that no person can affect their decision to take their ill child to health facilityand if present it was positive effect.

Table 1. Socio-demographic characteristics of sampledmothers, Khartoum ( $n=495)$.

\begin{tabular}{lll}
\hline Characteristics & Frequency & Percent \\
\hline Residence & & \\
Rural & 165 & $33 \%$ \\
Urban & 330 & $67 \%$ \\
Education level & & \\
Illiterate & 65 & $13.1 \%$ \\
Khalwa & 24 & $4.8 \%$ \\
Primary & 115 & $23.2 \%$ \\
Secondary & 188 & $38 \%$ \\
University & 94 & $19 \%$ \\
Postgraduate & 9 & $1.8 \%$ \\
Occupation & & \\
House wife & 376 & $76 \%$ \\
Employee & 65 & $13.1 \%$ \\
Temporal worker & 10 & $2 \%$ \\
Daily worker & 16 & $3.2 \%$ \\
Others & 28 & $5.7 \%$ \\
Income & & \\
Less than 500 & 65 & $13.1 \%$ \\
$500-1000$ & 115 & $23.2 \%$ \\
1001-1500 & 157 & $31.7 \%$ \\
More than 1500 & 156 & $31.5 \%$ \\
\hline
\end{tabular}

Table 2. Mothers response when their under five children get ill, Khartoum ( $n=495)$.

\begin{tabular}{lll}
\hline Characteristic & Frequency & Percent\% \\
\hline Did nothing & 36 & $7.3 \%$ \\
Went to the doctor directly & 348 & $70.3 \%$ \\
Went to the doctor after 24 hour & 48 & $9.7 \%$ \\
Went to the pharmacy & 21 & $4.2 \%$ \\
Went to traditional healer & 31 & $6.3 \%$ \\
Seek advice from family member & 3 & $0.6 \%$ \\
Others & 8 & $1.6 \%$ \\
Total & 495 & $100.0 \%$ \\
\hline
\end{tabular}


Table 3. Mothers action and residence, educational level, occupation, income, time work for facility and who took the decision of going to health facility.

\begin{tabular}{|c|c|c|c|c|}
\hline \multirow{2}{*}{ Residence } & \multicolumn{2}{|l|}{ Mothers action } & \multirow{2}{*}{$\mathbf{X}^{2}$} & \multirow{2}{*}{ P-value } \\
\hline & Seek health care & Not seek health care & & \\
\hline Urban & $255(77.3 \%)$ & $75(22.7)$ & \multirow{3}{*}{4.602} & \multirow{3}{*}{0.032} \\
\hline Rural & $141(85.5 \%)$ & $24(15.5 \%)$ & & \\
\hline Educational level & & & & \\
\hline Illiterate/ Khalwa & $72(80.9)$ & $13(20 \%)$ & \multirow{4}{*}{3.157} & \multirow{4}{*}{0.676} \\
\hline Primary /Secondary & $239(78.9 \%)$ & $64(21.1 \%)$ & & \\
\hline University/ Post graduate & $85(82.5 \%)$ & $18(17.5 \%)$ & & \\
\hline \multicolumn{3}{|l|}{ Occupation } & & \\
\hline House wife & $295(78.5 \%)$ & $81(21.5 \%)$ & \multirow{6}{*}{7.197} & \multirow{6}{*}{0.126} \\
\hline Employee & $59(90.8 \%)$ & $6(9.2 \%)$ & & \\
\hline Temporal worker & $9(90 \%)$ & $1(10 \%)$ & & \\
\hline Daily worker & $11(68.8 \%)$ & $5(31.2 \%)$ & & \\
\hline Others & $22(78.6 \%)$ & $6(21.4 \%)$ & & \\
\hline \multirow{2}{*}{\multicolumn{5}{|c|}{$\begin{array}{l}\text { Income } \\
\text { Less than } 500\end{array}$}} \\
\hline Less than 500 & $55(84.6 \%)$ & & & \\
\hline $500-1000$ & $87(75.7 \%)$ & $28(24.3 \%)$ & \multirow{3}{*}{10.686} & \multirow{3}{*}{0.030} \\
\hline $1001-1500$ & $129(82.2 \%)$ & $28(17.8 \%)$ & & \\
\hline More than 1500 & $125(80.1 \%)$ & $31(19.9 \%)$ & & \\
\hline \multicolumn{5}{|l|}{ Work time for health facility } \\
\hline For 24 hours & $160(75.1 \%)$ & $53(24.9 \%)$ & \multirow{3}{*}{5.571} & \multirow{3}{*}{0.018} \\
\hline Day time & $236(83.7 \%)$ & $46(16.3 \%)$ & & \\
\hline \multicolumn{3}{|l|}{ Who take the decision } & & \\
\hline Parents & $298(84.4 \%)$ & $55(15.6 \%)$ & & \\
\hline Mother & $76(75.2 \%)$ & $25(24.8 \%)$ & \multirow{4}{*}{30.492} & \multirow{4}{*}{0.000} \\
\hline Father & $20(60.6 \%)$ & $13(39.4 \%)$ & & \\
\hline Grandmother & $2(40 \%)$ & $3(60 \%)$ & & \\
\hline Others & $0(0 \%)$ & $3(100 \%)$ & & \\
\hline
\end{tabular}

Table 4. Logistic Regression for the factors affecting mothers'seeking behavior.

\begin{tabular}{|c|c|c|c|c|c|c|c|c|}
\hline \multirow{2}{*}{ Characteristics } & \multirow{2}{*}{ B } & \multirow{2}{*}{ S.E. } & \multirow{2}{*}{ Wald } & \multirow{2}{*}{ df } & \multirow{2}{*}{ Sig. } & \multirow{2}{*}{ Odd ratio } & \multicolumn{2}{|c|}{ 95\% C. I. for odd ratio } \\
\hline & & & & & & & Lower & Upper \\
\hline Residence & 0.585 & 0.296 & 3.921 & 1 & 0.048 & 1.795 & 1.006 & 3.204 \\
\hline Income & .098 & 0.135 & 0.531 & 1 & 0.466 & 1.103 & .847 & 1.436 \\
\hline Child age in months & $-0.027-$ & 0.010 & 6.914 & 1 & 0.009 & 0.974 & 0.954 & 0.993 \\
\hline Who takes the decision & 0.562 & 0.156 & 12.967 & 1 & 0.000 & 1.755 & 1.292 & 2.383 \\
\hline Work time for the facility & $-0.880-$ & 0.276 & 10.144 & 1 & 0.001 & 0.415 & 0.241 & 0.713 \\
\hline Services quality & $-0.357-$ & 0.181 & 3.877 & 1 & 0.049 & 0.700 & 0.491 & 0.998 \\
\hline Tradition and culture & $-0.796-$ & .286 & 7.739 & 1 & 0.005 & 0.451 & 0.258 & 0.790 \\
\hline Constant & 0.928 & 1.295 & 0.513 & 1 & 0.474 & 2.528 & & \\
\hline
\end{tabular}

\section{Discussion}

The multi-dimensional growth of a nation is primarily dependent on its health, peoplepower, and effective utilizationof services. It extends beyond the narrow limits of curative care, encompassing the preventive and promotiveaspects as well [8]. For effective health outcomes, it is mandatory that women utilize the services, because they are the prime caregivers to the children in the community.

MothersinKhartoum State have adequate health seeking behavior although considerable number did not seek care by doing nothing $(7.3 \%)$, going to the pharmacy directly $(4.2 \%)$ or going to traditional healers $(6.3 \%)$. Similar results were found in astudy conducted in western Sudan [9]. Where fever and malaria were defined correctly by the majority of caregivers and the majority of mothers seek health for their ill children. Nevertheless, contrast finding was found in a study conducted in Ethiopia, were less than half interviewed mothers seek health care for their ill children $(48.8 \%)$, of them only (27\%) seek health care within the first day of onset of symptoms [10]. High rate of utilization of public health facilities was found which might be attributable to the high level of awareness and coverage with health facilities, which indicated also through none association between disease severity and seeking health care.

Strong association between area of living and health seeking behavior was found, and this in accordance with a study conducted in Kenya [11]. On the other hand mother' education has been defined as an important predictor of health seeking behavior as many studies have discussed this issue [12]. The study revealed insignificant association between mother education and seeking health care, which can be explained by the approximately equal distribution of various levels of education among mothers. No significance association between mother'soccupation and seeking health. This finding is similar to study conducted in Nepal [13], where $83.3 \%$ mothers who worked outside home had sought treatment ( $\mathrm{p}=0.66)$. Regarding child' age, sex and order, the study revealed that significant association between children' 
age and seeking health care. This indicates that younger children have good attention by their mothers. The sex of child has no role according to thisstudy. This differs froma study in India which stated that there is a significant gender differential of healthcare use in rural India [14].

Income has significant relation with seeking health care. In India, a person's work status and monthly household income were significant explanatory variables for seeking care [15]. Giving the consideration of the fact that, the type of house and car owner-ship revealed as insignificant factors for not seeking care, income becomes the most prominent socioeconomic factor determining it. Availability of services and cost of services have no relation with mothers' seeking behavior in contrast to study conducted in Dhaka [16] where the cost of services is a predictor of seeking care. On the other hand, a study in Nicaragua [17] revealed that $70 \%$ of mothers have seek health care for their ill children and that was attributed to the low consultation cost of public health service, and partly attributable to the regularcommunity visits by government health staff members. Health insurance in recent years has improved the utilization of services and decreases the cost of services. Once family income is found as an important predictor for mother' seeking behavior, availability of health insurance for the family has significant relation with health seeking behavior. That means health insurance could improve health outcomes for children.

The study revealed that parents took the decision of taking their ill child to health facility by themselves which has strong association with positive health seeking behavior. In a study conducted in Nepal [13] thedecision for seeking treatment was made by husband of the respondentsin $46.4 \%$. Tradition and culture play major role in the mother' decision to seek health care for her ill child. The study revealed that a strong association between traditions and seeking behavior. This reflects deep effect of what have been said by elderly people.

Furthermore, time taken to reach the health facility and means of transportation have no relation with mothers' seeking behavior which indicates that mothers are not affected by external factors when their children get ill. On the other hand the study revealed that work time for the health facility and quality of services have significant relation with mother' seeking behavior, in accordance of study conducted in India where lack of accessibility, availability, and affordability of the healthcare services also was a cause, according to $68.3 \%$ of the mothers.

\section{Conclusions}

This study is conducted to identify, the frequency and factors affecting health seeking behavior among mothers of under five children residing in Khartoum State. Eighty percent of mothers seek health care for their children when being ill and bivariate and multivariate analysis revealed thatthe person who took the decision of going to health facility was the most predictor of mothers' seeking behavior, while tradition and culture found to be negatively associated with seeking care.

\section{Recommendations}

Progression towards health seeking behavior among mothersof under five children will be achieved byrising awareness among mothers about the importance of seeking health care for their ill children; health insurance coverage, improvement of the quality of health services at health facilities, and developing of clear policies for enhancement of seeking care.

\section{References}

[1] Ajibade B, Amoo P, Adeleke M, Oyadiran G, Kolade O, Olagunju R. Determinants of mothers health seeking behaviour for their children in a Nigerian teaching hospital. IOSR J Nurs Health Sci. 2013; 1: 9-16.

[2] Sudan health statistical report 2015.

[3] Campbell H, Duke $T$, Weber $M$, English $M$, Carai $S$, Tamburlini G. Global initiatives for improving hospital care for children: state of the art and future prospects. Pediatrics. 2008; 121 (4): e984-e92.

[4] Sudan Multiple Indicator Cluster Survey 2014.

[5] Mwenesi HA. Mothers' definition and treatment of childhood malaria on the Kenyan coast. 1994.

[6] Chandwani H, Pandor J. Healthcare-Seeking behaviors of mothers regarding their children in a tribal community of Gujarat, India. Electronic physician. 2015; 7 (1): 990.

[7] Ferdous F, Dil Farzana F, Ahmed S, Das SK, Malek MA, Das $\mathrm{J}$, et al. Mothers' perception and healthcare seeking behavior of pneumonia children in Rural Bangladesh. ISRN family medicine. 2014; 2014.

[8] Rosenstock IM. Why people use health services. The Milbank Quarterly. 2005; 83 (4).

[9] Webair HH, Bin-Gouth AS. Factors affecting health seeking behavior for common childhood illnesses in Yemen. Patient preference and adherence. 2013; 7: 1129.

[10] Simieneh MM, Mengistu MY, Gelagay AA, Gebeyehu MT. Mothers' health care seeking behavior and associated factors for common childhood illnesses, Northwest Ethiopia: community based cross-sectional study. BMC health services research. 2019; 19 (1): 59.

[11] Wambui WM, Kimani S, Odhiambo E. Determinants of Health Seeking Behavior among Caregivers of Infants Admitted with Acute Childhood Illnesses at Kenyatta National Hospital, Nairobi, Kenya. International journal of pediatrics. 2018; 2018

[12] Shaikh BT, Hatcher J. Health seeking behaviour and health service utilization in Pakistan: challenging the policy makers. Journal of public health. 2004; 27 (1): 49-54.

[13] Stekelenburg J, Jager BE, Kolk PR, Westen EH, van der Kwaak A, Wolffers IN. Health care seeking behaviour and utilisation of traditional healers in Kalabo, Zambia. Health policy. 2005; 71 (1): 67-81. 
[14] Sakisaka K, Jimba M, Hanada K. Changing poor mothers ' care-seeking behaviors in response to childhood illness: findings from a cross-sectional study in Granada, Nicaragua. BMC international health and human rights. 2010; 10 (1): 10.

[15] Taffa N, Chepngeno G. Determinants of health care seeking for childhood illnesses in Nairobi slums. Tropical Medicine \& International Health. 2005; 10 (3): 240-5.
[16] Wardlaw TM ,Johansson EW, Hodge MJ. Pneumonia: the forgotten killer of children: UNICEF; 2006.

[17] Shrestha P. Health Seeking Behavior among Mothers of Sick Children. Journal of Nepal Health Research Council. 2015. 